\title{
Design, Simulation, Fabrication and Characterization of Piezoelectric MEMS Cantilever for Gas Density and Viscosity Sensors Applications
}

\author{
A. Mehdaoui ${ }^{1}$, C.Huber ${ }^{1}$, J.Becker ${ }^{1}$, F.Schraner ${ }^{1}$, L.G.Villanueva ${ }^{2}$ \\ ${ }^{1}$ TrueDyne Sensors AG, Reinach BL, Switzerland \\ ${ }^{2}$ Ecole Polytechnique Fédérale de Lausanne, Advanced NEMS Laboratory, Lausanne, Switzerland \\ alexandre.mehdaoui@truedyne.com
}

\begin{abstract}
Summary:
A MEMS cantilever based resonant device for gas monitoring actuated and sensed piezoelectrically, has been designed, simulated, fabricated and tested. Aluminum Nitride (AIN) has been used as active material to implement the piezoelectric actuator and sensor. Simulation performed using COMSOL and measurements show a very good agreement. The final system, the full sensor for gas monitoring, allows the measurement of gas density and viscosity at temperatures between 0 and $65{ }^{\circ} \mathrm{C}$ and pressures between 1 and 10 bar abs. with accuracies of $<0.03 \mathrm{~kg} / \mathrm{m}^{3}$ and $6 \%$ respectively. The second technological run has shown significant results at the same conditions, respectively of $<0.02 \mathrm{~kg} / \mathrm{m}^{3}$ and $<2 \%$ for density and viscosity.
\end{abstract}

Keywords: MEMS, resonator, cantilever, piezoelectric actuation, piezoelectric sensing, density, viscosity, gas monitoring

\section{Background, Motivation an Objective}

Micromachined sensors are widely used to meet the increasing demand for miniaturized sensors for measuring physical parameters of gases, such as the density and viscosity. Also the realisation of ultra-precise scales in the field of inert gases e.g. welding gas or modified atmospheric packaging gas mixing application is of high interest. The use of standard silicon technology allows miniaturization at reduced costs, thus pushing the entry into new sensor markets such as low-power handheld systems [1]. The core of the sensor is an oscillating micro-cantilever which is fabricated at the Center of MicroNanotechnology (EPFL). The peak resonance response frequency $f_{r}$ and the quality factor $Q$ of a microcantilever are the two main dynamic characteristics that are very sensitive to the density and viscosity of the surrounding fluid [2]. Therefore, the viscosity and density of a fluid can be determined by analyzing the frequency response of a cantilever immersed in the fluid $[3,4]$.

\section{Description of the System: Design\&Process}

In this paper, a MEMS cantilever resonator (the core of the sensor), actuated and sensed piezoelectrically, has been designed, simulated, fabricated and tested. A piezoelectric transduction, Aluminum Nitride (AIN), is integrated on top of the cantilever to enable actuation and detection of devices (resonance frequency and $\mathrm{Q}$ factor). Platinum ( $\mathrm{Pt}$ ) has been used as top and bottom metal contacts between which AIN has been sandwiched to form the actuating and sensing electrodes and also the temperature sensor. Indeed, as the temperature has to be well measured and controlled during the evaluation of the gas thermophysical properties, we need to define the temperature sensor as close as possible of the density and viscosity sensors. The cantilever investigated in this paper was fabricated on a SOI wafer with a $10 \mu \mathrm{m}$ thick device layer and with a length of $L=600$ $\mu \mathrm{m}$ and a width of $W=202 \mu \mathrm{m}$. Fig. 1 shows the detailed process flow.

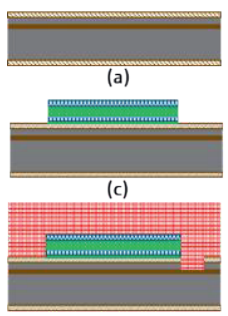

(e)
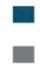

\$

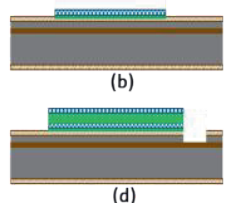

(d)
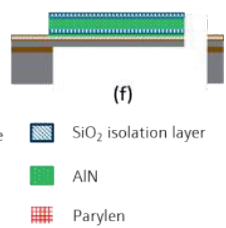

Fig. 1. AIN cantilever fabrication process: (a) thermal oxidation isolation layer, (b) adhesion AIN layer and bottom Pt metal deposition and patterned by liftoff, (c) active AIN layer and top Pt metal deposition 
by sputtering and patterned by $\mathrm{Cl}_{2} / \mathrm{Ar}$ dry etch, (d) cantilever shape patterning by $\mathrm{CF} 4$ and $\mathrm{Cl}_{2}$ dry etch, (e) Parylen deposition for the front side protection during the deep back side etching, (f) cavity patterning by dry $\mathrm{Si}$ and wet $\mathrm{SiO}_{2}$ etching, cantilever release by plasma $\mathrm{O}_{2}$

\section{Results}

Fig. 2a shows the SEM image of the fabricated AIN cantilever. Fig. $2 b$ shows the MEMS chip bonded on a PCB, following by the glob-top encapsulation.

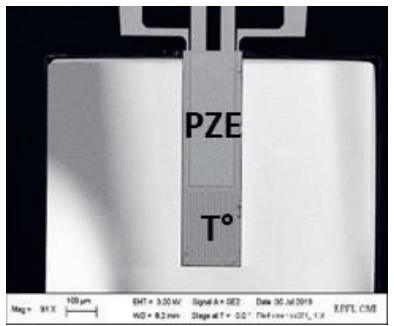

(a)

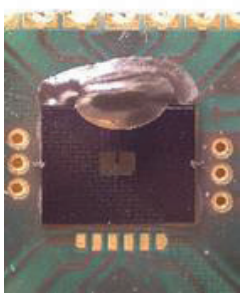

b)
Fig. 2. MEMS Piezoelectric cantilever: (a) SEM image: Temperature sensor $\left(T^{\circ}\right)$ and Piezoelectric transducer (PZE), (b) MEMS sample + PCB

Fig. $3 \mathrm{a}$ and $\mathrm{b}$ show respectively the simulated impedance real and imaginary parts performed in COMSOL and the measured frequency response, both amplitude and phase of the signal. We can see a good agreement between the simulation $\left(f_{r}=43.7 \mathrm{kHz}, \mathrm{Q}=882\right)$ and measurement results $\left(f_{r}=41.1 \mathrm{kHz}, Q=724\right)$.

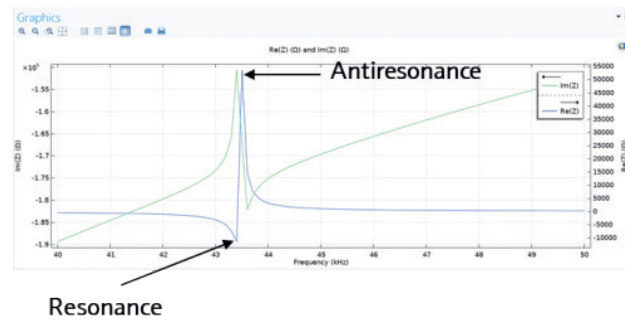

(a)

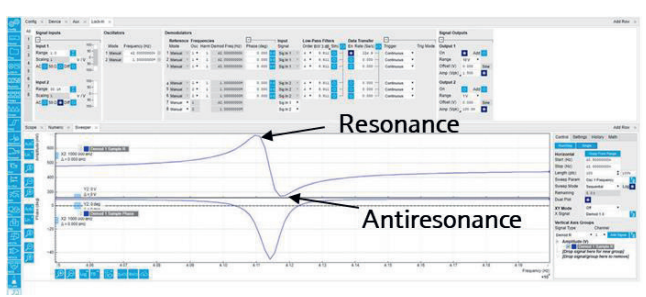

(b)

Fig. 3. Piezoelectric resonance and $Q$ factor in Air: (a) simulated impedance in COMSOL, (b) Measured frequency response using lock-in amplifier

For the measurement in gases, we have manufactured a special PCB containing a MEMS chip, a pressure and temperature sensors. This PCB can be screwed into a gas tight metal cyl- inder that serves as a gas cell, see Fig. 4. For a standard density and viscosity calibration, 4 different gases $\left(\mathrm{N}_{2}, \mathrm{CO}_{2}, \mathrm{Ar}\right.$ and $\left.\mathrm{He}\right)$ are measured at temperatures between 0 and $60{ }^{\circ} \mathrm{C}$ and pressures between 1 and 10 bar abs. We can measure the gas density with an abs. accuracy $<0.03 \mathrm{~kg} / \mathrm{m}^{3}$ and the dynamic viscosity with a relative accuracy of $6 \%$. The second technological RUN has shown better accuracies, respectively of $<0.02 \mathrm{~kg} / \mathrm{m}^{3}$ and $<2 \%$ for density and viscosity, by changing the Si device layer thickness from $10 \mu \mathrm{m}$ to $5 \mu \mathrm{m}$ and $3 \mu \mathrm{m}$. The measuring performance of the final sensor is shown in Fig. 5.

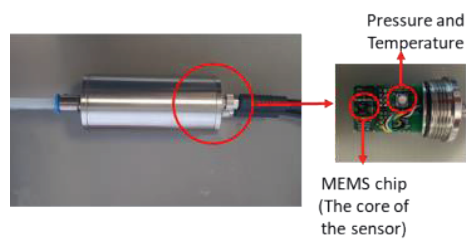

Fig. 4. Sensor PCB mounted in a pressure tight gas cell with electric connections on the right and fluidic connections on the left

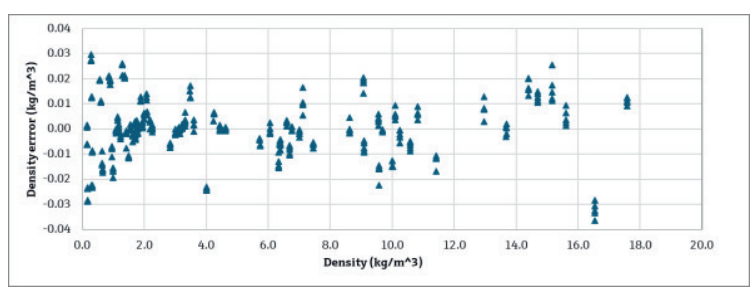

(a)

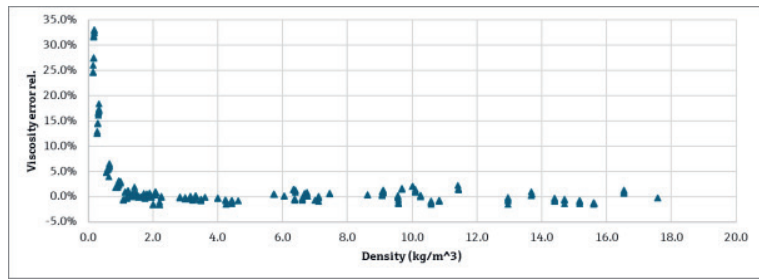

(b)

Fig. 5. (a) Density and (b) viscosity measuring accuracy

\section{References}

[1] M. Kucera and all, Characterization of multi roof tileshaped out of plane vibrational modes in aluminum nitride actuated self-sensing micro-resonators in liquid media, Applied Physics Letters 107, 053506053506 (2015), doi: 10.1063/1.4928429

[2] H. Qiu et al., Hydrodynamic analysis of piezoelectric microcantilevers vibrating in viscous compressible gases, Sensors and Actuators A, 238 (2016), 299-306

[3] A. Badarlis et al., Measurement and evaluation of the gas density and viscosity of pure gases and mixtures using a micro-cantilever beam, Sensors, 15 (2016), doi: $10.3390 / \mathrm{s} 150924318$

[4] C. Huber et al., Gas density and viscosity measurement with a micro-cantilever for online combustion gas monitoring, 19. GMA/ITG-Fachtagung Sensoren and Messsysteme, 2018 